\title{
Automated Diagnosis Non-proliferative Diabetic Retinopathy in Fundus Images using Support Vector Machine
}

\author{
Raju Sahebrao \\ Maher \\ Department of \\ Computer Science\& IT \\ Dr. Babasaheb \\ Ambedkar Marathwada \\ University \\ Aurangabad, India
}

\author{
Sangramsing N. \\ Kayte \\ Department of \\ Computer Science\& IT \\ Dr. Babasaheb \\ Ambedkar Marathwada \\ University \\ Aurangabad, India
}

\author{
Sandip T. Meldhe \\ S.D.Deshmukh College \\ Bhokardan \\ Dr. Babasaheb \\ Ambedkar Marathwada \\ University \\ Aurangabad, \\ Maharashtra, India
}

\author{
Mukta \\ Dhopeshwarkar, \\ $\mathrm{PhD}$ \\ Department of \\ Computer Science\& IT \\ Dr. Babasaheb \\ Ambedkar Marathwada \\ University \\ Aurangabad, India
}

\begin{abstract}
Diabetic retinopathy (DR) is caused by damage the retina because fluid leaks from blood vessels into the retina. Damage the posterior part of the eye of the diabetic patient. This disease that occurs when does not secrete enough insulin or the body is unable to process it properly. The main two types of diabetic retinopathy the first are non-proliferate diabetes retinopathy (NPDR) and second are proliferate diabetes retinopathy (PDR). The increasing number of DR cases world-wide demands to the development of an automated detection system. We have proposed a computer based method for the detection of diabetic retinopathy using the fundus images. Using Image pre-processing, morphological processing techniques involves processing of fundus images for detect features, such as blood vessel area, exudates, microaneurysms, hemorrhages, and texture. Proposed techniques used for the extraction of these features from digital fundus images. The proposed techniques have been tested on the images of DIARETDB0 database. In which have total 130 images they all images are tested and it's classified into microaneurysms, hemorrhages, and texture using Support Vector Machine (SVM) for an automatic classification. The detection results obtained by comparing it with expert ophthalmologists. We demonstrated a classification sensitivity of $96.43 \%$, specificity of $95.9 \%$ and accuracy of $99.27 \%$.
\end{abstract}

\section{Keywords}

Diabetic Retinopathy, Fundus images, Microaneurysms, Exudates, Retinal blood vessels. Image morphology, artificial neural network.

\section{INTRODUCTION}

Diabetic retinopathy is a complication of diabetes and a leading cause of blindness in the world. It occurs when diabetes damages the tiny blood vessels inside the retina. This tiny blood vessel will leak blood and fluid on the retina retinopathy are occur such as microaneurysms, hemorrhages, hard exudates, cotton wool spots. The number of people afflicted with the disease continues to grow it. Blindness is an effect of diabetic retinopathy and its prevalence is set to continue rising. Estimated 50-65 new cases of blindness per 100,000 people happened every year [1]. The World Health Organization (WHO) expects the number of people with diabetics to increase from 130 million to 350 million over the next 25 years. [1]. Diabetic retinopathy (DR) is a common complication of diabetes. Indeed, it is so common that it is the leading cause of blindness in the working population of western countries [2]. Diabetes is increasing in developed countries, as well as in underdeveloped countries. It is estimated that $75 \%$ of people with diabetic retinopathy live in developing countries [3]. Diabetes Retinopathy is a silent disease, because it may only be recognized by the patient when the changes in the vision. Diabetic Retinopathy has been proved to $17 \%$ to $97 \%$ of the cases after 5 and 15 years of the diagnosis of diabetes respectively. It have progressed to a level where treatment is difficult and nearly impossible. The impairment of vision and blindness can be prohibited or detected if accordingly regular screening and treated of eyes. Automated detection will lead to a large amount of savings of time and effort. In recent years, the growing of number of diabetic patients has greatly motivated the research work in automatic developing tools and methodologies to facilitate the screening problems.

This paper main proposes an automated technique for microaneurysms and hemorrhages in retinal images using an image pre-processing techniques such as thresholding and morphological reconstruc-tion, and boundary sketching to detect the dark lesions, such as microaneurysms and hemorrhages using a SVM classifier to classify the retinal images into normal and abnormal (i.e. NPDR and PDR). The NPDR severity scale is further classified as mild and moderate. RGB image is convert in the green channel image good contrast between the background and the dark lesions retinal components, such as microaneurysms and hemorrhages, it is reliable to work on the green channel of the RGB color space in order to detect the dark objects easily. The proposed classification algorithm is classify the various disease.

\subsection{Diabetes}

Diabetes mellitus (DM) is the name of a chronic, systemic, life-threatening disease. Metabolism refers to the way our bodies use digested food for energy and growth. Most of what we eat is broken down into glucose. Glucose is a form of sugar in the blood - it is the principal source of fuel for our bodies. It occurs when the pancreas does not secrete enough insulin or the body is unable to process it properly. This results in an abnormal increase in the glucose level in the blood. Over time this high level of glucose causes damage to blood vessels. This damage affects both eyes and nervous 
system, as well as heart, kidneys and other organs [4].The diabetes have two types. The first is Diabetes type 1 results from a failure of the human body to produce insulin. Type 1 diabetes is less common than another type of diabetes type 2 . People with type 1 diabetes take insulin injections. Near about $81 \%$ of people with type 2 diabetes are overweight. That is part of a metabolic syndrome in that includes obesity, elevated blood pressure, and high levels of blood lipids.

\subsection{Diabetes Retinopathy}

Diabetic retinopathy is a complicated of diabetes mellitus. Diabetic retinopathy is the most normal cause of new cases of blindness among adults aged $20-70$ years. During the first two periods of disease, nearly all patients with type 1 diabetes and greater than $90 \%$ of patients with type 2 diabetes have retinopathy. Diabetes affects the eyes by causing corrosion of blood vessels in the retina. Collapse of retinal blood vessels may result in fluid leaking into the center of the retina (macular edema) or abnormal blood vessels that grow on the surface of the retina (neovascularization) which can bleed and scar. This can lead to loss of central and possibly peripheral vision. In the younger-onset group, $86 \%$ of blindness was attributable to diabetic retinopathy. In the older-onset group, in which other eye diseases were common, one-third of the cases of legal blindness were due to diabetic retinopathy [5]. Diabetic retinopathy progresses from mild nonproliferative abnormalities, charac-terized by increased vascular permeability, to moderate and severe nonproliferative diabetic retinopathy (NPDR), characterized by vascular closure, to proliferative diabetic retinopathy (PDR), characterized by the growth of new blood vessels on the retina and posterior surface of the vitreous. Macular edema, characterized by retinal thickening from leaky blood vessels, can develop at all stages of retinopathy. Pregnancy, puberty, blood glucose control, hypertension, and cataract surgery can accelerate these changes. Types of diabetic retinopathy DR can be broadly classified as non-proliferative DR (NPDR) and proliferative DR (PDR) depending on the presence of specific DR features, the stages can be identified.

\subsection{Non-proliferative diabetic retinopathy}

Non-proliferative diabetic retinopathy is the earlier stage of diabetic retinopathy is characterized by visible damage to small retinal blood vessels. These blood vessels swelling called microaneurysms. Micro-aneurysms and other areas of abnormal retinal blood vessels may leak fluid, causing the retina to bleed or swell. This may lead to vision loss. Leakage in the center of the retina, known as macular edema, is the most common mechanism of vision loss in people with diabetic retinopathy. Non-proliferative diabetic retinopathy is the most common form of diabetic retinopathy, accounting for approximately $80 \%$ of all cases.

\section{- Microaneurysms}

The primary stage of abnormality that occurs in the eye due to DR is the Microaneurysm. These are identified as tiny, dark red spots or miniscule haemorrhages, either appearing alone or in clusters, inherent to the light sensitive retina. The Microaneurysm is circular in shape and the size varies from 10-100 microns i.e. 1/12th the diameter of an average optics disc. The disease is not alarming at this phase.

\section{- Haemorrhages}

These are also termed 'blot' haemorrhages, with regard to their round shape. These are found in the deeper layers of the retina.

\section{- Hard exudates}

The hard exudates are found in diverse sizes from puny blots to booming tracts with clear peripheries and these are the vital symptoms of Diabetic Retinopathy. Commonly the eye encompasses a fluid that is rich in fat and protein alongside blood, which oozes out from the exudates. Such a phenomenon prevents light from reaching the retina thereby leading to visual impairment.

\section{- $\quad$ Soft exudates}

In extreme stages of Diabetic Retinopathy, certain spots called the 'cotton wool spots' are identified. The retinal pre capillary arterioles supplying blood to the nerve fiber layer are clogged and associatively the local nerve fiber axons.

\subsection{Proliferative diabetic retinopathy}

Proliferative Retinopathy stage is advanced stage, in this stage the signals sent by the retina for nourishment trigger the growth of new blood vessels. These new blood vessels are abnormal. Proliferative diabetic retinopathy can also lead to traction retinal detachments. They grow along the retina and along the surface of the clear, vitreous gel that fills the inside of the eye. The retinal neovascularization can grow to be large and then contract, pull, and lift the retina. These blood vessels do not cause symptoms or vision loss. However, they have thin and they leak blood, severe vision loss and even blindness can result. Retinal detachment can also lead to loss of vision if it involves the macula.

This paper proposed is using the proposed methods for detection of microaneurysms and hemorrhages, emphasizing its main characteristics and common problems in the area. Details information of the method for detection of microaneurysms and hemorrhages and the results achieved by the proposed method and compares these results with other methods in the literature; and, the finally presents the conclusion of this paper.

\section{MATERIALS AND METHODS}

The detection method proposed in this paper is performed in MATLAB (version 2012). The accuracy of the method was tested in the public database of fundus images DIARETDB1 database consists of 89 colour fundus images of which 84 contain at least mild non-proliferative signs of the diabetic retinopathy and five are considered as normal which do not contain any signs of the diabetic retinopathy according to all the experts participated in the evaluation. Images were captured with the same 50 degree FOV digital fundus camera with varying imaging controlled by the system in the Kuopio university hospital, Finland. The image ground truth provided along with the database is based on expert selected findings related to the diabetic retinopathy and normal fundus structures.

\subsection{Pre- processing}

In pre-processing, we create binary masks for background and noisy areas. Different retinal images make it difficult to extract retinal features and distinguish exudates from other contrast, and brightness bright features in images. The enhancement technique is applied at first so that the result is more suitable in terms of usefulness for further analysis than the original image. Histogram equalisation in which manipulates histograms in a consis-tent and meaningful manner. DIARETDB1 images were resized to $640 \mathrm{x} 480$ pixels in order to reduce the processing time of the method However, many image enhan-cement techniques are based on spatial operations performed on local neighbour-hoods of 
input pixels. Such poor quality as to interfere with analysis. In approximately $10 \%$ of the retinal images, artefacts are significant enough to impede human grading.

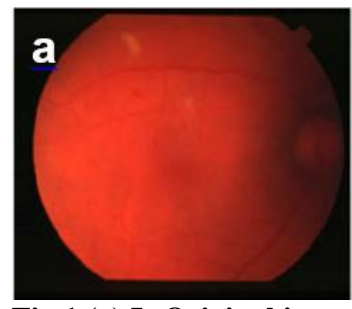

Fig.1 (a) Is Original image

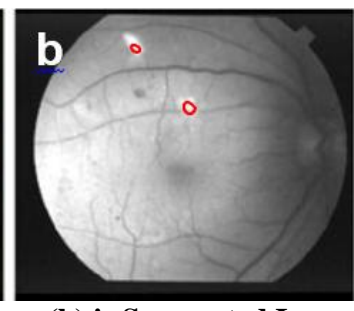

(b) is Segmented Image
Pre-processing of such images can ensure adequate level of success in the automated abnormality detection. In the retinal images there can be variations caused by the factors including differences in cameras, illumination, acquisition angle and retinal pigmentation.

\subsection{The Proposed System}

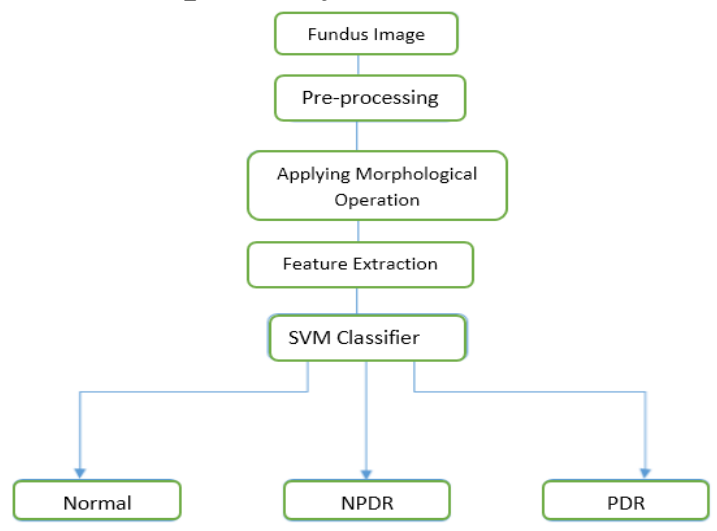

Fig.2 Proposed decision support framework for NPDR classification

First step in the pre-processing is to attenuate such image variations by normalizing the colour of the original retinal image against a reference image. Few of the retinal images acquired using standard clinical protocols often exhibit low contrast. Also, retinal images typically have a higher contrast in the centre of the image with reduced contrast moving outward from the centre. Images, a local contrast enhancement method is applied as a second preprocessing step. Finally it is required to create a fundus mask for each image to facilitate segmentation of lesion.

\subsection{Detection of blood vessels}

The detection of the blood vessels was implemented using the morphological opening process to that proposed in the blood vessels have a linear feature. The length of the structuring element was defined with the value 40 pixels, so that the openings do not consider rounded structures that could be associated with the red lesions. Automated detection system is developed to detect retinal blood vessels Gabor filter. The area of lesions and texture features are then used to construct a feature vector that is input to the multiclass support vector machine (SVM) for classifying images into normal, mild, moderate, severe and proliferative categories [20]. Finally, the 12 images obtained were added, getting the image, containing a sketch of the blood vessels, according to Equalization.

The segmentation of blood vessels in colour retinal images using Gabor filter. The problem with blood vessel segmentation is that the visibility of vascular pattern is usually not good especially for thin vessels. So, it is neces-sary to enhance the vascular pattern. It was found that the appearance of vessels is highly sensitive in the grey scale image containing only the wavelength of green channel. Area and perimeter calculated from the RGB components of the blood vessels were used as features to classify normal, mild, moderate, severe and proliferative stages of retinopathy using a feed forward neural network [6]. For segmentation of vessels was performed using only green channel of RGB colour image. Gabor filter, whose application can be found in problems such as, strokes in character recognition and detecting roads in satellite image analysis, were explored to detect and enhance vessel features in retinal image. When compared with the matched filter for detecting line like features, Gabor filter provided a better result as it has optimal localization in both the frequency and space domains.

The Gabor filter was tuned to a suitable frequency and orientation was able to emphasize vessels along that direction. Values of all the filter parameter were selected based on the properties of vessels. When filter was aligned along orientation of vessel it produced single peak response along that direction. Gabor filters oriented along different directions in the range of 0 to 170 degrees were used to enhance the multi-oriented vessels. The resulted enhanced vessels were then subjected to thresholding for vessel pixel classification. Entropic threshold calculation based on grey level co-occurrence matrix as it contained information on the distribution of grey level frequency and edge information have been presented.

\section{RESULTS AND DISCUSSIONS}

The two standard diabetic retinopathy fundus image databases are. The DIARETDB0 database consists of 130 color fundus images of which 20 are normal and 110 contain signs of the DR (MA,HE and Cotton Wools Spots)[26]. The DIARETDB1 database consists of 89 color fundus images of which 84 contain at least mild NPDR signs (MA's and HE) of the DR, and 5 are considered as normal which do not contain any signs of the DR according to all experts who participated in the evaluation [27]. In this database contains 130 fundus images while DIARETDB1 database contains 89 retinal images.

DIARETDB0 and DIARETDB1 These databases contain overall 219 retinal images with a resolution of $1500 \times 1152$ pixels and of different qualities in terms of noise and illumination. Images were captured using the same $150^{\circ} \mathrm{FOV}$ digital fundus camera with varying imaging settings. Figures 2 the output of classifier highlighting dark and bright lesions from retinal images respectively.

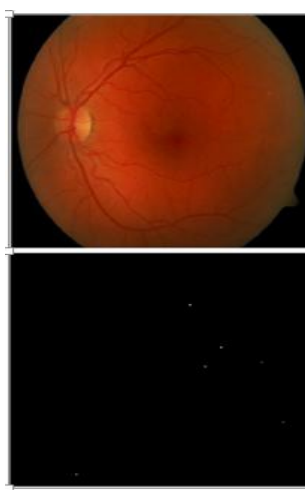

(a)

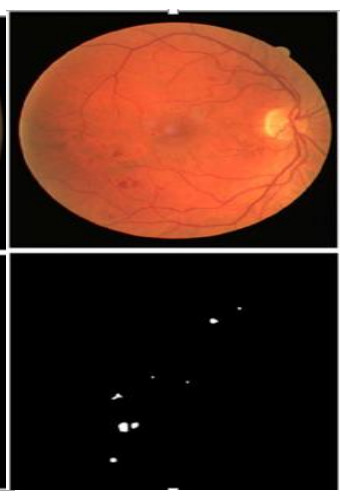

(b)
Fig.2 A. Original Image and Detection of HE B. Original Image and Dark lesions segmentation [13] 
A total of 60 images are selected from all two databases at random and for those images, a comparison between proposed method and ground truth for lesion. The performance of the proposed system is evaluated on the basis of four measures, namely, True positive (TP) indicates that the patient suffers from the disease and test result was also positive, false positive (FP) indicates that the patient does not suffer from the disease and was diagnosed as positive, true negative (TN) indicates that the patient does not suffer from the disease and was diagnosed as negative, false negative (FN) indicates that the patient suffers from the disease but diagnosed as negative. TP represents the fraction of pixels correctly classified as bright lesion pixels. This measure is also known as sensitivity [10].There calculating are following equations.

$$
\text { Sensitivity }=\frac{\text { TP }}{\mathrm{TP}+\mathrm{FN}} \times 100
$$

Specificity is defined as the percentage of normal images classified by the system Accuracy in percentage is the measure of

$$
\text { Specificity }=\frac{\mathrm{TN}}{\mathrm{TN}+\mathrm{FP}} \times 100
$$

Accuracy is the percentage of correctly classified normal and abnormal images

$$
\text { Accuracy }=\frac{\mathrm{TP}+\mathrm{TN}}{(\mathrm{TP}+\mathrm{FN}+\mathrm{TN}+\mathrm{FP})} \times 100
$$

Table 1: Comparison of vessel segmentation results on DIARETDB0 \& DIARETDB1 and other database

\begin{tabular}{|c|c|c|c|}
\hline Method & $\begin{array}{c}\text { Sensitivity } \\
(\mathbf{\%})\end{array}$ & $\begin{array}{c}\text { Specificity } \\
(\mathbf{\%})\end{array}$ & $\begin{array}{c}\text { Accuracy } \\
(\mathbf{\%})\end{array}$ \\
\hline Walter [10] & $92.8 \%$ & $92.4 \%$ & $100 \%$ \\
\hline Wasif [11] & $94.3 \%$ & $92.0 \%$ & $100 \%$ \\
\hline $\begin{array}{c}\text { Proposed } \\
\text { method }\end{array}$ & $96.43 \%$ & $95.9 \%$ & $99.27 \%$ \\
\hline
\end{tabular}

The proposed technique achieved a Sensitivity for DIARETDB0 and DIARETDB1 databases of $95.97 \%$ and $96.90 \%$ respectively and overall $96.43 \%$ for bright lesions (MA \& HE). The comparisons of proposed technique with already published methods are performed and summarized in Tables the comparison of sensitivity and accuracy for dark lesions detection the results for bright lesions detection.

\section{CONCLUSIONS AND FUTURE WORK}

An automated system for diabetic retinopathy detection has been presented. In this work we have investigated and proposed a computer-based system extracts blood vessels and exudates for grading the severity of the diabetic retinopathy to identify normal, NPDR and PDR. Early detection of diabetic retinopathy is very important because it enables timely treatment that can ease detect bright objects sharply with an average sensitivity $96.43 \%$. The experimental results show that the proposed method yields better sensitivity and predictive values compared to other methods. Therefore, one of the major strengths of the proposed system is accurate feature extractions and accurate grading of NPDR lesions. In this paper image processing components required to build an automated system for early detection of diabetic retinopathy.

\section{REFERENCES}

[1] World Diabetes, A newsletter from the World Health Organization, 4, 1998.

[2] Ong, G. L., Ripley, L. G., Newsom, R. S., Cooper, M., and Casswell, A. G., Screening for sight-threatening diabetic retinopathy: comparison of fundus photography with automated color contrast threshold test. Am. J. Ophthalmol. 137(3):445-452, 2004.

[3] Orbis. Retrieved from: http://www.orbis. org. Last accessed December 2009.

[4] Alberti, K. G., and Zimmet, P. Z., Definition, diagnosis and classification of diabetes mellitus and its complications, part 1: diagnosis and classification of diabetes mellitus provisional report of a WHO consultation. Diabet. Med. 15(7):539-553, 1998.

[5] UK Prospective Diabetes Study Group: Tight blood pressure control and risk of macrovascular and microvascular complications in type 2 diabetes: UKPDS 38.BMJ 317: 708-713, 1998.

[6] Wong, L. Y., Acharya, U. R., Venkatesh, Y. V., Chee, C., Lim, C. M., and Ng, E. Y. K., Identification of different stages of diabetic retinopathy using retinal optical images. Information Sciences 178(1):106-121, 2008.

[7] [20]. Adarsh. P and D. Jeyakumari, Multiclass SVMBased Automated Diagnosis of Diabetic Retinopathy International conference on Communication and Signal Processing, April 3-5, 2013, India.

[8] 26.Kauppi, T., Kalesnykiene, V., Kamarainen, J. K., Lensu, L., Sorri, I., Uusitalo, H., Kälviäinen, H., Pietilä, J., DIARETDB0: Evaluation database and methodology for diabetic retinopathy algorithms. Technical Report, 2006.

[9] Kauppi, T., Kalesnykiene, V., Kamarainen, J.-K., Lensu, L., Sorri, I., Raninen A., Voutilainen R., Uusitalo, H., Kälviäinen, H., Pietilä, J., DIARETDB1 diabetic retinopathy database and evaluation protocol, Technical report, 2007.

[10] Walter, T., Klein, J.-C., Massin, P., and Erginay, A., A contribution of image processing to the diagnosis of diabetic retinopathy-detection of exudates in color fundus images of the human retina. IEEE Trans. Med. Imag. 21 (10)1236-1243, 2002.

[11] Reza, A. W., Eswaran, C., and Hati, S., Automatic tracing of optic disc and exudates from color fundus images using fixed and variable thresholds. J. Med. Syst. 33 (1) $73-80,2009$.

[12] Raju Maher, Sangramsing Kayte, Dr. Mukta Dhopeshwarkar "Review of Automated Detection for Diabetes Retinopathy Using Fundus Images" International Journal of Advanced Research in Computer Science and Software Engineering Volume 5, Issue 3, March 2015 